\title{
DHEA Supplementation Confers No Additional Benefit to that of Growth Hormone on Pregnancy and Live Birth Rates in IVF Patients Categorized as Poor Prognosis
}

\author{
Kevin N. Keane ${ }^{1,2 t}$, Peter M. Hinchliffe ${ }^{2}$, Philip K. Rowlands'2, Gayatri Borude ${ }^{2}$, \\ Shanti Srinivasan ${ }^{2}$, Satvinder S. Dhaliwal ${ }^{3}$ and John L. Yovich ${ }^{1,2 *+}$ \\ ${ }^{1}$ School of Pharmacy and Biomedical Science, Faculty of Health Sciences, Curtin University, Perth, WA, Australia, ${ }^{2}$ PIVET Medical \\ Centre, Perth, WA, Australia, ${ }^{3}$ Faculty of Health Sciences, School of Public Health, Curtin University, Perth, WA, Australia
}

OPEN ACCESS

Edited by:

Michael Von Wolff,

University Hospital Bern,

Switzerland

Reviewed by:

Matheus Roque,

ORIGEN - Center for

Reproductive Medicine,

Brazil

Leif Johan Bungum,

IVI Middle East Fertility Clinic LLC,

United Arab Emirates

*Correspondence:

John L. Yovich

jlyovich@pivet.com.au

tThese authors have contributed equally to this work.

Specialty section: This article was submitted to Reproduction,

a section of the journal

Frontiers in Endocrinology

Received: 11 October 2017 Accepted: 12 January 2018

Published: 31 January 2018

Citation:

Keane KN, Hinchliffe PM,

Rowlands PK, Borude G,

Srinivasan S, Dhaliwal SS and

Yovich JL (2018) DHEA

Supplementation Confers No Additional Benefit to that of Growth Hormone on Pregnancy and Live

Birth Rates in IVF Patients Categorized as Poor Prognosis.

Front. Endocrinol. 9:14. doi: 10.3389/fendo.2018.00014
Background: In vitro fertilization (IVF) patients receive various adjuvant therapies to enhance success rates, but the true benefit is actively debated. Growth hormone (GH) and dehydroepiandrosterone (DHEA) supplementation were assessed in women undergoing fresh IVF transfer cycles and categorized as poor prognosis from five criteria.

Methods: Data were retrospectively analyzed from 626 women undergoing 626 IVF cycles, where they received no adjuvant, GH alone, or GH-DHEA in combination. A small group received DHEA alone. The utilization of adjuvants was decided between the attending clinician and the patient depending on various factors including cost.

Results: Despite patients being significantly older with lower ovarian reserve, live birth rates were significantly greater with $\mathrm{GH}$ alone (18.6\%) and with GH-DHEA (13.0\%) in comparison to those with no adjuvant $(p<0.003)$. No significant difference was observed between the $\mathrm{GH}$ groups $(p=0.181)$. Overall, patient age, quality of the transferred embryo, and GH treatment were the only significant independent predictors of live birth chance. Following adjustment for patient age, antral follicle count, and quality of transferred embryo, GH alone and GH-DHEA led to a 7.1-fold and 5.6-fold increase in live birth chance, respectively $(p<0.000)$.

Conclusion: These data indicated that GH adjuvant may support more live births, particularly in younger women, and importantly, the positive effects of $\mathrm{GH}$ treatment were still observed even if DHEA was also used in combination. However, supplementation with DHEA did not indicate any potentiating benefit or modify the effects of GH treatment. Due to the retrospective design, and the risk of a selection bias, caution is advised in the interpretation of the data.

Keywords: growth hormone, dehydroepiandrosterone, in vitro fertilization, embryo quality, adjuvants

\section{INTRODUCTION}

Assisted reproductive technology (ART) incorporating in vitro fertilization (IVF) are key strategies to increase the possibility of conceiving for individuals who have experienced difficulties with natural conception. As a result of socioeconomic factors in both Western and Asian cultures, there is an increasing propensity for males and females to delay childbirth until an older age. Due to the 
natural process of decreased fertility with age, this has meant that increasing numbers of patients are seeking support from fertility specialists. In addition, with the global increase in endocrine disorders including obesity and metabolic syndrome, along with other ovarian pathologies that affect fertility, for instance endometriosis, adenomyosis and leiomyomata, patient management is challenged over and above the impact of increasing age. Historically, a subgroup of patients defined as poor prognosis according to the Bologna criteria (1) have proved very difficult to treat and have poor outcomes. They possess a combination of characteristics such as advanced maternal age (40 years and above), poor response to standard gonadotropin stimulation (less than four oocytes on collection), and/or a minimal ovarian reserve, as evident by a low anti-Mullerian hormone (AMH) level (less than $0.5-1.1 \mathrm{ng} / \mathrm{ml} ; 3.7-8.1 \mathrm{nmol} / \mathrm{l}$ ) or low antral follicle count (AFC; less than 5-7 follicles). However, these criteria do not include patients who produce poor quality embryos for unknown reasons and are equally considered poor prognosis. Nonetheless, ART clinics are faced with an increased demand for improved treatment, and one mechanism to address this, but which has proved highly controversial, has been to include offlabel adjuvant therapy as a part of IVF management.

Two of the most common IVF adjuvant therapies are growth hormone $(\mathrm{GH})$ and dehydroepiandrosterone (DHEA) supplementation (2). However, as a consequence of various investigations that have been either poorly designed or utilized small patient cohorts, along with a lack of sufficiently powered randomized controlled trials (RCTs) in this area, the true beneficial effects of GH and DHEA are actively debated and still remain clinically unclear (2). GH is an anabolic peptide hormone that functions to increase cell proliferation and growth, and it has been demonstrated to improve the sensitivity to gonadotropin stimulation and boost oocyte yield in several IVF studies (3-9). Recently, we (10) along with others $(9,11-15)$ found that GH promoted pregnancy rate and/or live birth chance by reducing miscarriage rates $(13,16,17)$. It is hypothesized that $\mathrm{GH}$ reduces aneuploidies by enhancing embryo quality, and some evidence indicated that it may have a role in this respect (14), but the molecular mechanism is not understood. On the other hand, numerous other studies $(5,18-23)$, along with some meta-analyses and systematic reviews $(7,8)$, have demonstrated that $\mathrm{GH}$ has no clear positive effect on pregnancy or live birth outcomes in IVF patients. Similarly, DHEA, a multifunctional adrenal prohormone that acts as a precursor for testosterone and estradiol synthesis (24), has also been reported to promote enhanced pregnancy and live birth rates in poor prognosis IVF patients (25-34). In addition, DHEA has been suggested to increase fertilization rates and embryo quality $(27,32$, $35,36)$. Equally, other investigations including meta-analyses $(15,37-39)$ have demonstrated no clear benefit in terms of oocytes collected, embryo quality, or clinical pregnancy and live birth rates $(30,40-43)$. Thus, the use of these adjuvant therapies in IVF remains highly controversial and inconclusive (2).

These hormones are believed to modulate ovarian physiology, including oocyte and follicle maturation, and could have local effects on the endometrium during ovulation and implantation $(37,38,44)$. To date, very little research, RCT or observational, has investigated the synergistic effects of $\mathrm{GH}$ and DHEA in combination in the IVF setting (45). Following on from our previous 2017 retrospective investigation where we found that $\mathrm{GH}$ in isolation enhanced clinical outcomes (10), we aimed to determine whether the addition of DHEA to treatment cycles resulted in any further beneficial outcomes. Consequently, in this study, we examined the effect of $(+) \mathrm{GH}$ alone, (+)DHEA alone, and $(+) \mathrm{GH}-\mathrm{DHEA}$ in combination on poor prognosis IVF patients and primary outcomes included pregnancy and live birth rates. As a secondary aim, due to the controversy surrounding the use of IVF adjuvants and the definition of poor prognosis patients, we intended to identify significant variables that should be considered when designing future prospective studies in this research area. Therefore, we included a broader definition of "poor prognosis" IVF patients, which extended the Bologna criteria to those with repetitive implantation failure (RIF) and those who generated lower quality embryos.

\section{MATERIALS AND METHODS}

\section{Study Period and Participants}

This retrospective study covered a period from 1 April 2008 to 31 December 2015 and is subsequent to another study investigating GH administration in isolation (10). The current analysis specifically focused on a subset of patients who were offered IVF adjuvants at any point in their treatment history by attending clinicians, because they were classified as poor prognosis with one or more of the following criteria: (i) women with fewer than four metaphase II (M II) oocytes although receiving maximal FSH stimulation (i.e., $450 \mathrm{IU} /$ day); (ii) women where the majority of embryos were graded poor quality with marked fragmentation (>50\%) (46); (iii) women with repetitive fresh or frozen embryo transfers ( $\geq 3$ transfers) without pregnancy; (iv) women aged $\geq 40$ years who had at least one failed IVF cycle; and (v) women with $\leq 8$ antral follicles. The data collection, storage, and analysis were conducted independently from the clinicians who conducted the consultations, IVF procedures, and prescribed adjuvants.

To limit the bias of including multiple treatment cycles for individual patients and in an attempt to randomize cases, only the first IVF cycle with successful ovum pick-up (OPU) and fresh embryo transfer (ET) for each patient during the study period was included in the analysis. Furthermore, only cycles where either no adjuvant therapy, GH-only, DHEA-only, or GH and DHEA in combination was administered were included. This meant that cycles with other adjuvant such as melatonin were excluded. In total, 626 eligible women had 626 IVF cycles that resulted in successful OPU and fresh ET. A total of 239 cycles/ women had their first cycle within the time period that was free from any adjuvant intervention, designated (-)Adj. A total of 161 different women had 161 cycles with GH alone $(+) \mathrm{GH}$, while 42 others received DHEA alone (+)DHEA, and 184 received $\mathrm{GH}$ and DHEA in combination (+)GH-DHEA (Figure 1). Each cycle/woman included in the analysis represented their first initiated IVF cycle within the study period but not necessarily their first in their treatment history. Patients elected to use GH, DHEA, 


\section{All Initiated Cycles \\ I $^{\text {st }}$ April $2008-3$ | $^{\text {st }}$ December 2015}

$(n=3505)$

$\downarrow$

All Cycles from Patients who were

offered adjuvants

$(n=\mid 488)$

Exciuding $(n=440) \ldots$

Donor Cycles $(n=33)$

Cancelled Cycles $(n=137)$

Failed Fertilisation $(n=102)$ Failed

All Cycles with Fresh Embryo Transfer TVOA $(n=49)$

Ectopic, Blighted Ovum $(n=\mid I)$

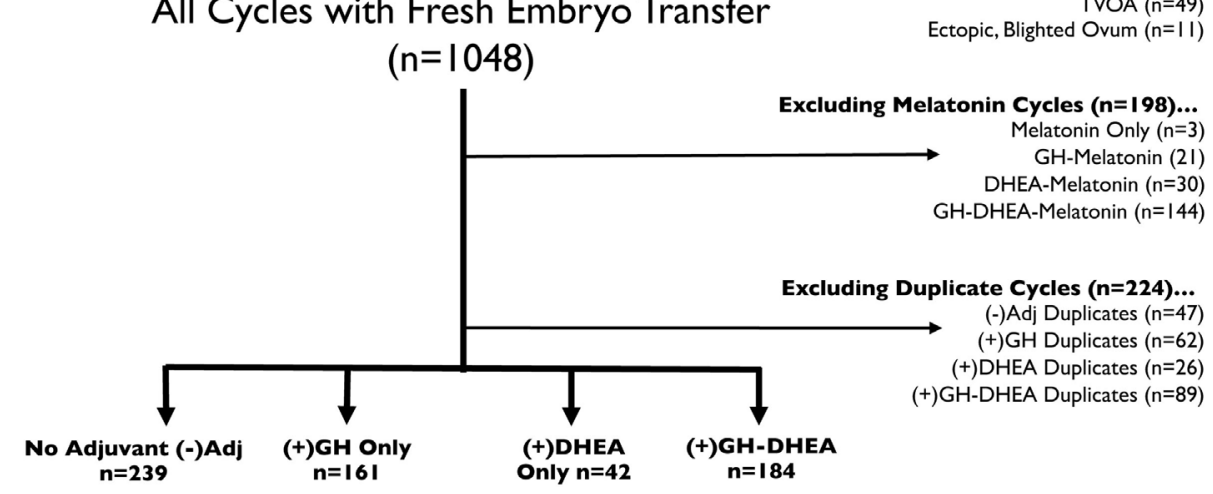

FIGURE 1 | Flow diagram of data extraction. Data were extracted from the PIVET database, and cases/cycles removed on the basis of cycle outcome (e.g. canceled/donor), melatonin treatment, and cycle type (failed transvaginal oocyte aspiration, failed fertilization, or freeze all).

or the combination based on the several factors, one of which was expense (since patients were charged for the drugs).

\section{Clinical Management}

Growth hormone (Saizen) was administered during the preceding menstrual cycle commencing on day $2-3$, thereafter the injection of six vials of 9 IU over 6 weeks in the lead-up to OPU, and equated to $54 \mathrm{IU}$ over 33-37 days averaging approximately $1.5 \mathrm{IU}$ per day. SciTropin $(0.3 \mathrm{mg})$ was injected daily for 45 days prior to trigger, with patients receiving $\mathrm{GH}$ at precisely $1.0 \mathrm{IU}$ per day up to OPU (10). For DHEA administration, each patient utilized one half of a DHEA troche containing $25 \mathrm{mg}$ of micronised DHEA sublingual twice daily, commencing in the preceding menstrual cycle and given over a period of 6 weeks in the lead-up to OPU (47). Where GH was administered in combination with DHEA, the two adjuvants were given over the same 6-week period.

All patients were stimulated with recombinant FSH using specific dosage algorithms as defined recently (48) and in most cases (43.9\% of cycles) using an antagonist protocol (Orgalutran). Older women with a low AFC rating received a flare-agonist regimen $(37.1 \%)$ or specialized downregulation protocols $(19.0 \%)$ (49) (Table 1). These stimulation protocols applying the PIVET dosing algorithm have been well described with minimized OHSS risk and fewer than $4 \%$ of cases overall generating more than 20 oocytes (48). Ovulation was triggered with human chorionic gonadotrophin (HCG). OPU was performed using transvaginal oocyte aspiration and undertaken $36 \mathrm{~h}$ posttrigger with IV sedation and using a double-lumen flushing/aspiration needle (Cook, Australia). The luteal phase was managed using HCG support (50). Additional support hormones were given as required (estradiol, progesterone, or combined estradiol/ progesterone pessary). Where $\geq 12$ oocytes were recovered, progesterone pessaries replaced HCG injections.

\section{Embryo Culture and Assessment}

Retrieved oocytes were cultured for $4-5 \mathrm{~h}$ postcollection prior to insemination with spermatozoa $(100,000 / \mathrm{ml})$ for IVF, or denuded with hyaluronidase and mature oocytes were injected using ICSI. Day 3 embryos were graded using a four-point system, with half points increments as previously published (51). Day 5 embryos were graded using the Gardner scoring system for blastocysts (52). While the clinic has a strong policy for single embryo transfer, patients categorized as poor prognosis can receive up to two day 3 embryos and such ensued in $85(-)$ Adj, 93 (+)GH, 18 (+)DHEA, and 93 (+)GH-DHEA cycles, respectively. On a very rare occasion, three day 3 embryos were transferred and occurred in one cycle each for $(-) \mathrm{Adj},(+) \mathrm{GH}$, and $(+) \mathrm{GH}-\mathrm{DHEA}$ treatment. Blastocysts were transferred in a minority of these poor prognosis cycles $(9.9 \%)$ as the clinic policy requires $>3$ high-quality embryos progressing on cleavage stage day 3 to allow culture through to day 5 or day 6 .

\section{Data Analysis and Statistics}

Primary clinical outcomes included likelihood of clinical pregnancy (presence of an intrauterine gestational sac with 
TABLE 1 | Overview of main parameters that affect clinical pregnancy and live birth rates.

\begin{tabular}{|c|c|c|c|c|c|}
\hline & \multirow{2}{*}{$\begin{array}{l}\text { No clinical } \\
\text { pregnancy }\end{array}$} & \multicolumn{2}{|c|}{ Yes clinical pregnancy } & \multirow[t]{2}{*}{ Totals } & \multirow[t]{2}{*}{$p$ value } \\
\hline & & No live birth & Yes live birth & & \\
\hline Number of cycles & 534 & 27 & 65 & 626 & \\
\hline Age, years (SD) & $39.0 \pm 4.2$ & $38.3 \pm 3.7$ & $36.6 \pm 4.1^{\mathrm{a}}$ & - & $<0.000$ \\
\hline $\mathrm{AMH}, \mathrm{pmol} / \mathrm{I}(\mathrm{SD})$ & $8.0 \pm 9.8$ & $9.0 \pm 11.3$ & $10.5 \pm 12.7$ & - & 0.521 \\
\hline $\mathrm{BMl}, \mathrm{kg} / \mathrm{m}^{2}(\mathrm{SD})$ & $24.2 \pm 4.8$ & $26.8 \pm 4.9^{\mathrm{a}}$ & $24.5 \pm 4.2$ & - & 0.017 \\
\hline Embryos transferred, N (SD) & $1.46 \pm 0.51$ & $1.52 \pm 0.51$ & $1.52 \pm 0.50$ & - & 0.589 \\
\hline Oocytes retrieved, N (SD) & $6.8 \pm 4.4$ & $7.6 \pm 5.0$ & $8.0 \pm 4.6$ & - & 0.078 \\
\hline Oocyte utilization rate, \% (SD) & $40.6 \pm 25.5$ & $39.7 \pm 26.9$ & $36.5 \pm 21.1$ & - & 0.458 \\
\hline Two pronuclei generated, N (SD) & $3.7 \pm 2.7$ & $4.6 \pm 3.0$ & $4.8 \pm 3.4^{\mathrm{a}}$ & - & 0.002 \\
\hline Fertilization rate, \% (SD) & $59.0 \pm 23.7$ & $62.3 \pm 23.9$ & $60.8 \pm 19.1$ & - & 0.683 \\
\hline Embryos cryopreserved, N (SD) & $0.59 \pm 1.15$ & $0.81 \pm 1.24$ & $0.86 \pm 1.33$ & - & 0.154 \\
\hline Embryo utilization rate, \% (SD) & $69.4 \pm 30.7$ & $63.2 \pm 29.6$ & $61.7 \pm 29.5$ & - & 0.107 \\
\hline Percentage of high-quality embryos, \% (SD) & $33.3 \pm 26.6$ & $39.0 \pm 29.3$ & $43.2 \pm 23.0^{a}$ & - & 0.012 \\
\hline Percentage of medium-quality embryos, \% (SD) & $39.5 \pm 24.4$ & $34.8 \pm 23.6$ & $38.2 \pm 20.5$ & - & 0.579 \\
\hline Percentage of low-quality embryos, \% (SD) & $27.2 \pm 25.8$ & $26.2 \pm 28.7$ & $18.6 \pm 16.5^{a}$ & - & 0.034 \\
\hline \multicolumn{6}{|l|}{ Age groups } \\
\hline$<35$ years & $85(15.9 \%)$ & $3(11.1 \%)$ & $20(30.8 \%)$ & 108 & 0.001 \\
\hline 35-39 years & $170(31.8 \%)$ & $14(51.9 \%)$ & $28(43.1 \%)$ & 212 & \\
\hline 40-44 years & $253(47.4 \%)$ & $10(37.0 \%)$ & $17(26.2 \%)$ & 280 & \\
\hline$>44$ years & $26(4.9 \%)$ & $0(0.0 \%)$ & $0(0.0 \%)$ & 26 & \\
\hline \multicolumn{6}{|l|}{ AFC groups } \\
\hline A (>20 follicles) & $56(10.5 \%)$ & $5(18.5 \%)$ & $9(13.8 \%)$ & 70 & 0.035 \\
\hline B (13-19 follicles) & 78 (14.6\%) & $2(7.4 \%)$ & $11(16.9 \%)$ & 91 & \\
\hline C (9-12 follicles) & 95 (17.8\%) & 8 (29.6\%) & $14(21.5 \%)$ & 117 & \\
\hline D (5-8 follicles) & 192 (36.0\%) & $10(37.0 \%)$ & $25(38.5 \%)$ & 227 & \\
\hline$E(<5$ follicles $)$ & $113(21.2 \%)$ & $2(7.4 \%)$ & $6(9.2 \%)$ & 121 & \\
\hline \multicolumn{6}{|l|}{ Stimulation groups } \\
\hline Antagonist & $234(43.8 \%)$ & $10(37.0 \%)$ & $31(47.7 \%)$ & 275 & 0.532 \\
\hline Flare agonist & 198 (37.1\%) & $9(33.3 \%)$ & 25 (38.5\%) & 232 & \\
\hline Other (downregulation) & $102(19.1 \%)$ & $8(29.6 \%)$ & $9(13.8 \%)$ & 119 & \\
\hline \multicolumn{6}{|l|}{ Adjuvant groups } \\
\hline$(-)$ Adj & 215 (40.3\%) & $13(48.1 \%)$ & $11(16.9 \%)$ & 239 & 0.118 \\
\hline$(+) \mathrm{GH}$ & $126(23.6 \%)$ & 5 (18.5\%) & $30(46.2 \%)$ & 161 & \\
\hline$(+) \mathrm{DHEA}$ & 39 (7.3\%) & $3(11.1 \%)$ & $0(0.0 \%)$ & 42 & \\
\hline (+)GH-DHEA & 154 (28.8\%) & $6(22.2 \%)$ & 24 (36.9\%) & 184 & \\
\hline
\end{tabular}

aStatistically different from no clinical pregnancy group.

Patient age was the most significant predictor of successful clinical pregnancy or live birth rates. Also patients with a larger proportion of high-quality embryos had greater live birth rates. Key parameters such as $A M H$ level, AFC, and stimulation protocol did not alter these rates.

AFC, antral follicle count; $A M H$, anti-Mullerian hormone; BMI, body mass index; DHEA, dehydroepiandrosterone; GH, growth hormone.

fetal heart beat at 7 weeks gestation) and/or live birth. Logistic regression was used to assess the independent contributions of individual confounding parameters on these outcomes such as age, body mass index (BMI), AMH level, AFC, stimulation protocol type, quality, developmental stage, and number of embryos transferred, in addition to the number of patient infertility factors and the number of previous IVF attempts. The unadjusted effect of adjuvant administration on these binary outcomes was also assessed. The effect of each variable was expressed as an odds ratio (OR) with associated 95\% confidence interval (CI). Stepwise multiple logistic regression analyses enabled the determination of the minimum number of independent variables that could be used for predicting clinical pregnancy and/or live birth chance. The coefficients of the independent variables from each of the final logistic regression models were used to calculate OR and CI for clinical pregnancy and/or live birth chance due to adjuvant treatment. Continuous variables were compared using two-sample $t$-tests, and categorical variables were compared using Fisher's Exact Chi-square tests.

\section{RESULTS \\ Overview of Patient Demographics and Adjuvant Treatment Groups}

The majority of the cycles analyzed in this poor prognosis cohort resulted in no clinical pregnancy (85.3\%), as expected for a poor prognosis cohort. The overall pregnancy rate was $14.7 \%$, whereas the live birth rate was $10.4 \%$ (miscarriage rate of $29.3 \%$, 27/92; Table 1). The majority of women were aged between 35 and 44 years (78.6\%), with an AFC of $\leq 8$ follicles $(348 / 626$; $55.6 \%$ ), and most received antagonist stimulation (43.9\%). For those who became pregnant, they tended to have more embryos cryopreserved (mean of 0.8 versus 0.6 embryos) and were significantly younger (mean age of 37.1 versus 39.0 years), generating significantly more high-quality embryos (42.0\% versus $33.3 \%$ ) 
and zygotes with two pronuclei (4.8 versus 3.7 zygotes). These trends were more pronounced for those who went on to have a successful live birth. There was no significant difference in the mean number of embryos transferred, mean oocytes retrieved, or oocyte/embryo utilization rates among those who failed to become pregnant, those who did become pregnant, those who miscarried, and those who had a live birth (Table 1).

No adjuvant (-)Adj, was administered in $38.2 \%$ of analyzed cycles, while $(+) \mathrm{GH},(+)$ DHEA and $(+) \mathrm{GH}-$ DHEA was used in $25.7,6.7$, and $29.4 \%$ of cycles, respectively (Table 2 ). However, in all of the cycles where there was a live birth, $83.1 \%$ were derived from a (+)GH cycle [i.e., (+)GH and (+)GH-DHEA]. Only $16.9 \%$ of live births came from (-)Adj cycles. The largest proportion of live birth (46.2\%) were from (+)GH alone cycles. Addition of DHEA did not alter this rate significantly with $36.9 \%$ of live births from cycles with GH and DHEA in combination. However, no live births were recorded in the small (+)DHEA-only group of 42 cases (Table 1). The majority of miscarriages occurred in (-) Adj cycles (48.1\%; Table 1). Overall, the clinical pregnancy rate in $(-) \mathrm{Adj},(+) \mathrm{GH},(+) \mathrm{DHEA}$, and (+)GH-DHEA groups was 10.0, $21.7,7.1$, and $16.3 \%$, respectively, and the live birth rate was 4.6 , 18.6, 0.0, and $13.0 \%$, respectively (Table 2 ).

From the included patient cohort, there was no significant difference between the groups with regard to the mean BMI; fertilization rate; number of embryos cryopreserved; and proportion of high-, medium-, or low-quality embryos generated after OPU (Table 2). However, there were significant differences between the adjuvant treatment groups and (-)Adj for age [younger in (-) Adj], AMH [higher in (-)Adj], mean embryos transferred [fewer in (-)Adj], mean oocytes retrieved [more in (-)Adj], mean two pronuclei generated [more in (-)Adj], and utilization rates [lower in (-)Adj; Table 2].

\section{Univariate and Multivariate Analysis Using Logistic Regression}

Logistic regression models were generated to determine the influence of each individual variable on clinical pregnancy and live birth ORs. Only patient age, transferred embryo development stage (blastocyst versus cleavage stage), transferred embryo quality, and the adjuvant treatment type were significant predictors of clinical pregnancy and/or live birth chance. AFC only affected pregnancy rates significantly in the lower AFC category $(\leq 4$ follicles). Patient AMH, BMI, number of embryos transferred, stimulation protocol type, infertility factors, or previous IVF attempts did not influence clinical pregnancy and/or live birth chance significantly or independently (Table 3). When stepwise multiple logistic regression was performed using all terms, only patient age, transferred embryo quality, and adjuvant treatment type were retained and were each independently significant; thus, they were the most important parameters for primary outcome prediction. AFC was not significant for clinical pregnancy or live birth chance in the multivariable model. Overall, increasing patient age decreased the chance of clinical pregnancy and/or live birth by about $10 \%$ per advancing year. Following adjustment for age, AFC, and adjuvant treatment type, transferred embryo quality was an independent predictor of clinical pregnancy and live birth, with greatest ORs observed when high-quality day 3 or high-/ medium-quality blastocysts were transferred (Table 3). Adjuvant treatment type was also an independent predictor of clinical pregnancy and live birth chance following adjustment for patient age, AFC, and transferred embryo quality, with the largest ORs observed for $(+) \mathrm{GH}[\mathrm{OR}, 3.28(p<0.000)$ and $7.07(p<0.000)$ for pregnancy and live birth, respectively]. Conversely, (+)DHEA alone did not alter the chance of clinical pregnancy or live birth, with (-)Adj as a reference comparator. Addition of DHEA to

TABLE 2 | Overview of main parameters for adjuvant treatment groups.

\begin{tabular}{|c|c|c|c|c|c|}
\hline & (-)Adj & $(+) \mathrm{GH}$ & (+)DHEA & (+)GH-DHEA & $p$ value \\
\hline Number of cycles & 239 & 161 & 42 & 184 & - \\
\hline Age, years (SD) & $37.5 \pm 4.3$ & $39.1 \pm 4.1^{\mathrm{a}}$ & $39.2 \pm 4.1$ & $39.9 \pm 3.9^{a}$ & 0.000 \\
\hline $\mathrm{AMH}, \mathrm{pmol} / \mathrm{l}(\mathrm{SD})$ & $12.2 \pm 13.3$ & $5.3 \pm 6.3^{a}$ & $4.9 \pm 4.8^{\mathrm{a}}$ & $7.0 \pm 7.7^{\mathrm{a}}$ & 0.000 \\
\hline $\mathrm{BMI}, \mathrm{kg} / \mathrm{m}^{2}(\mathrm{SD})$ & $24.4 \pm 4.8$ & $24.2 \pm 5.5$ & $24.4 \pm 4.5$ & $24.3 \pm 4.4$ & 0.997 \\
\hline Embryos transferred, N (SD) & $1.36 \pm 0.49$ & $1.59 \pm 0.51^{\mathrm{a}}$ & $1.43 \pm 0.50$ & $1.52 \pm 0.51^{\mathrm{a}}$ & 0.000 \\
\hline Oocytes retrieved, N (SD) & $7.7 \pm 4.3$ & $7.2 \pm 5.1$ & $5.4 \pm 3.3^{a}$ & $6.2 \pm 4.4^{\mathrm{a}}$ & 0.000 \\
\hline Oocyte utilization rate, \% (SD) & $33.4 \pm 21.2$ & $44.4 \pm 27.0^{a}$ & $42.1 \pm 23.3$ & $44.5 \pm 26.9^{a}$ & 0.000 \\
\hline Two pronuclei generated, N (SD) & $4.2 \pm 2.9$ & $4.1 \pm 5.2$ & $3.2 \pm 2.1$ & $3.5 \pm 2.9^{a}$ & 0.036 \\
\hline Fertilization rate, \% (SD) & $56.6 \pm 22.9$ & $58.9 \pm 23.1$ & $64.2 \pm 23.9$ & $62.2 \pm 23.5$ & 0.067 \\
\hline Embryos cryopreserved, N (SD) & $0.72 \pm 1.22$ & $0.73 \pm 1.29$ & $0.38 \pm 0.73$ & $0.49 \pm 1.07$ & 0.076 \\
\hline Embryo utilization rate, \% (SD) & $61.4 \pm 30.2$ & $74.5 \pm 33.0^{a}$ & $68.8 \pm 28.3$ & $71.4 \pm 28.4^{\mathrm{a}}$ & 0.000 \\
\hline Percentage of high-quality embryos, \% (SD) & $32.9 \pm 24.5$ & $37.0 \pm 27.4$ & $37.7 \pm 24.6$ & $34.0 \pm 28.6$ & 0.403 \\
\hline Percentage of medium-quality embryos, \% (SD) & $41.3 \pm 22.5$ & $38.5 \pm 24.7$ & $36.8 \pm 26.0$ & $37.1 \pm 24.8$ & 0.303 \\
\hline Percentage of low-quality embryos, \% (SD) & $25.8 \pm 23.2$ & $24.2 \pm 25.1$ & $25.5 \pm 25.6$ & $28.9 \pm 27.6$ & 0.360 \\
\hline Fresh embryo transfer cycles, $\mathrm{N}$ & 239 & 161 & 42 & 184 & - \\
\hline Fresh ET pregnancy rate, N (\%) & 24/239 (10.0) & $35 / 161(21.7)$ & $3 / 42(7.1)$ & 30/184 (16.3) & 0.005 \\
\hline Fresh ET live birth rate, N (\%) & $11 / 239(4.6)$ & 30/161 (18.6) & $0 / 42(0.0)$ & 24/184 (13.0) & 0.000 \\
\hline Fresh ET miscarriage rate, $\mathrm{N}(\%)$ & $13 / 24(54.2)$ & $5 / 35(14.3)$ & 3/3 (100.0) & $6 / 30(20.0)$ & 0.000 \\
\hline
\end{tabular}

a Statistically different from (-)Adj group.

From the complete data set, there was no significant difference between (+)GH cycles and (-)GH with regard to patient BMI; mean fertilization rate; proportion of low-, medium-, or high-quality embryos generated; mean number of oocytes retrieved; or mean number of zygotes generated with two pronuclei. However, despite the (+)GH and (+)GH-DHEA groups being significantly older and having a significantly lower AMH in comparison to the (-)Adj group, they achieved greater oocyte and embryo utilization rates.

AMH, anti-Mullerian hormone; BMI, body mass index; DHEA, dehydroepiandrosterone; ET, embryo transfer; GH, growth hormone. 
TABLE 3 | Logistic regression analysis of cycles.

\begin{tabular}{|c|c|c|c|c|c|c|c|c|}
\hline \multirow[t]{2}{*}{ Variable } & \multicolumn{4}{|c|}{ Clinical pregnancy chance } & \multicolumn{4}{|c|}{ Live birth chance } \\
\hline & $\begin{array}{l}\text { Univariate } \\
\text { (unadjusted) }\end{array}$ & $p$ value & Multivariable & $p$ value & $\begin{array}{l}\text { Univariate } \\
\text { (unadjusted) }\end{array}$ & $p$ value & Multivariable & $p$ value \\
\hline \multicolumn{9}{|l|}{ Adjuvant type } \\
\hline$(-) \operatorname{Adj}$ & 1.00 & - & 1.00 & - & 1.00 & - & 1.00 & - \\
\hline$(+) \mathrm{GH}$ & $2.49(1.42-4.37)$ & 0.002 & $3.28(1.78-6.01)$ & 0.000 & 4.75 (2.30-9.79) & 0.000 & 7.07 (3.24-15.41) & 0.000 \\
\hline$(+) \mathrm{DHEA}$ & $0.69(0.20-2.40)$ & 0.559 & $0.96(0.27-3.47)$ & 0.950 & $\mathrm{NC}$ & $\mathrm{NC}$ & $\mathrm{NC}$ & NC \\
\hline$(+) \mathrm{GH}-\mathrm{DHEA}$ & $1.75(0.98-3.10)$ & 0.058 & $2.89(1.54-5.42)$ & 0.001 & $3.11(1.48-6.53)$ & 0.003 & $5.64(2.52-12.64)$ & 0.000 \\
\hline Age & $0.90(0.86-0.95)$ & 0.000 & $0.90(0.85-0.95)$ & 0.000 & 0.89 (0.84-0.94) & 0.000 & $0.86(0.81-0.92)$ & 0.000 \\
\hline Serum AMH & $1.02(0.99-1.05)$ & 0.291 & - & - & $1.02(0.98-1.06)$ & 0.286 & - & - \\
\hline BMl & 1.05 (1.00-1.10) & 0.056 & - & - & $1.01(0.96-1.07)$ & 0.716 & - & - \\
\hline Number of embryos transferred & $1.26(0.81-1.94)$ & 0.303 & - & - & $1.25(0.76-2.06)$ & 0.386 & - & - \\
\hline \multicolumn{9}{|l|}{ AFC grouping (follicle) } \\
\hline$A(\geq 20)$ & 1.00 & - & 1.00 & - & 1.00 & - & 1.00 & - \\
\hline B (13-19) & 0.67 (0.29-1.53) & 0.338 & $0.86(0.35-2.12)$ & 0.749 & $0.93(0.36-2.39)$ & 0.883 & $1.20(0.42-3.40)$ & 0.739 \\
\hline $\mathrm{C}(9-12)$ & $0.93(0.44-1.96)$ & 0.841 & $1.44(0.61-3.37)$ & 0.405 & $0.92(0.38-2.26)$ & 0.857 & $1.15(0.40-3.28)$ & 0.791 \\
\hline$D(5-8)$ & $0.73(0.37-1.45)$ & 0.368 & $1.32(0.59-2.95)$ & 0.502 & $0.84(0.37-1.90)$ & 0.672 & $1.43(0.55-3.72)$ & 0.466 \\
\hline$E(\leq 4)$ & $0.28(0.11-0.72)$ & 0.008 & $0.57(0.20-1.61)$ & 0.290 & $0.35(0.12-1.04)$ & 0.059 & $0.71(0.21-2.42)$ & 0.587 \\
\hline \multicolumn{9}{|l|}{ Stimulation protocol } \\
\hline Antagonist cycle & 1.00 & - & - & - & 1.00 & - & - & - \\
\hline Agonist cycle & $0.98(0.60-1.60)$ & 0.936 & - & - & $0.95(0.54-1.66)$ & 0.859 & - & - \\
\hline Other cycle (downregulation) & $0.95(0.52-1.75)$ & 0.873 & - & - & $0.64(0.30-1.40)$ & 0.266 & - & - \\
\hline \multicolumn{9}{|l|}{ Blastocyst versus cleavage } \\
\hline Cleavage & 1.00 & - & - & - & 1.00 & - & - & - \\
\hline Blastocyst & $3.25(1.81-5.85)$ & 0.000 & - & - & $2.93(1.51-5.68)$ & 0.001 & - & - \\
\hline \multicolumn{9}{|l|}{ Quality of transferred embryo } \\
\hline Low-quality D3 & 1.00 & - & 1.00 & - & 1.00 & - & 1.00 & - \\
\hline High-quality blastocyst & $5.55(2.33-13.21)$ & 0.000 & $5.67(2.06-15.60)$ & 0.001 & $3.75(1.26-10.98)$ & 0.017 & $3.19(0.89-11.50)$ & 0.076 \\
\hline Medium-quality blastocyst & $3.54(1.29-9.72)$ & 0.014 & $3.55(1.21-10.46)$ & 0.021 & $4.82(1.6014 .55)$ & 0.005 & $5.26(1.54-17.97)$ & 0.008 \\
\hline Low-quality blastocyst & $3.14(0.81-12.25)$ & 0.099 & $2.08(0.47-9.17)$ & 0.334 & $5.46(1.36-21.95)$ & 0.017 & $3.36(0.69-16.38)$ & 0.134 \\
\hline High-quality D3 & $1.90(1.15-3.13)$ & 0.012 & $1.80(1.06-3.06)$ & 0.030 & $2.48(1.36-4.52)$ & 0.003 & $2.45(1.28-4.70)$ & 0.007 \\
\hline \multicolumn{9}{|l|}{ Number of infertility factors } \\
\hline None or one factor & 1.00 & - & - & - & 1.00 & - & - & - \\
\hline Two factors & $0.66(0.41-1.04)$ & 0.075 & - & - & $0.72(0.42-1.23)$ & 0.228 & - & - \\
\hline Three or more factors & $0.61(0.27-1.38)$ & 0.235 & - & - & $0.70(0.28-1.76)$ & 0.445 & - & - \\
\hline \multicolumn{9}{|l|}{ Number of previous IVF attempts } \\
\hline No previous attempts & 1.00 & - & - & - & 1.00 & - & - & - \\
\hline One previous attempts & $1.37(0.82-2.32)$ & 0.233 & - & - & $1.00(0.54-1.83)$ & 0.994 & - & - \\
\hline Two previous attempts & $1.51(0.73-3.11)$ & 0.268 & - & - & $0.86(0.34-2.18)$ & 0.750 & - & - \\
\hline Three or more previous attempts & $1.25(0.63-2.46)$ & 0.525 & - & - & $1.32(0.64-2.73)$ & 0.460 & - & - \\
\hline
\end{tabular}

AFC, antral follicle count; AMH, anti-Mullerian hormone; BMI, body mass index; DHEA, dehydroepiandrosterone; GH, growth hormone; IVF, in vitro fertilization; NC, not computed due to low case number.

The presence of GH, patient age, transferred embryo development stage, and quality were the only significant variables that affected clinical pregnancy or live birth chance. When adjusting for these variables in a multivariate logistic analysis, the effect of each parameter became stronger, as reflected by increased odds ratios.

GH cycles did not alter the significance of (+)GH-DHEA ORs for clinical pregnancy or live births, with the positive effect from GH still observed [OR, $2.89(p=0.001)$ and $5.64(p<0.000)$ for pregnancy and live birth, respectively].

\section{Analysis According to Patient Age and Adjuvant Treatment}

Since the majority of patients (78.6\%) in this poor prognosis cohort were aged between 35 and 44 years, our analysis focused on two specific age groups, 35-39 years and 40-44 years (Table 4). In women aged 35-39 years, (+)GH led to significantly more clinical pregnancies and live births (OR, 4.50; 95\% CI, 1.81-11.15 and
$14.68,95 \%$ CI, 3.14-68.76, $p=0.001$, respectively). There was a trend for increased clinical pregnancies in the $(+)$ DHEA group (OR, 2.12; 95\% CI, $0.40-11.38 ; p=0.379$ ), but it was not significant. The case number leading to live births in this group was too few to analyze. However, the addition of DHEA in combination with GH did not alter the positive enhancement of clinical pregnancies and live births from GH (OR, 3.50; 95\% CI, 1.41-8.65 and OR, 15.50; $95 \% \mathrm{CI}, 3.37-71.28, p<0.007$, respectively). On the other hand, $(+) \mathrm{GH}$ was the only adjuvant group to demonstrate any advantage in women aged 40-44 years, showing that there was a significant increase in live birth chance (OR, 5.79; 95\% CI, 1.23-27.80; $p=0.027$; Table 4). This was reflected in the specific age range of 40-41 years (Table 4). 
TABLE 4 | Logistic regression analysis of age interaction with $\mathrm{GH}$.

\begin{tabular}{|c|c|c|c|c|c|c|c|}
\hline & $\begin{array}{c}\text { No clinical } \\
\text { pregnancy, } \mathrm{N}(\%)\end{array}$ & $\begin{array}{c}\text { Yes clinical } \\
\text { pregnancy, } \mathrm{N}(\%)\end{array}$ & $\begin{array}{l}\text { Clinical pregnancy, } \\
\text { odds ratio }(95 \% \mathrm{Cl})\end{array}$ & $p$ value & $\begin{array}{c}\text { Yes live } \\
\text { birth, } N(\%)\end{array}$ & $\begin{array}{l}\text { Live birth, odds } \\
\text { ratio }(95 \% \mathrm{Cl})\end{array}$ & $p$ value \\
\hline \multicolumn{8}{|l|}{ Unadjusted analysis } \\
\hline$(-) \mathrm{GH}, \mathrm{N}(\%)$ & $215(90.0)$ & $24(10.0)$ & 1.00 & - & $11(4.6)$ & 1.00 & - \\
\hline$(+) \mathrm{GH}, \mathrm{N}(\%)$ & $126(78.3)$ & $35(21.7)$ & $2.49(1.42-4.37)$ & 0.002 & $30(18.6)$ & $4.75(2.30-9.79)$ & 0.000 \\
\hline (+)DHEA, N (\%) & $39(92.8)$ & $3(7.1)$ & $0.69(0.20-2.40)$ & 0.559 & $0(0.0)$ & $\mathrm{NC}$ & $\mathrm{NC}$ \\
\hline$(+) \mathrm{GH}-\mathrm{DHEA}, \mathrm{N}(\%)$ & $154(83.7)$ & $30(16.3)$ & $1.75(0.98-3.10)$ & 0.058 & $24(13.0)$ & $3.11(1.48-6.53)$ & 0.003 \\
\hline \multicolumn{8}{|c|}{ Analysis according to age group } \\
\hline \multicolumn{8}{|l|}{ Age $<35$ years } \\
\hline$(-) \mathrm{GH}, \mathrm{N}(\%)$ & $47(82.5)$ & $10(17.5)$ & 1.00 & - & 7 (12.3) & 1.00 & - \\
\hline$(+) \mathrm{GH}, \mathrm{N}(\%)$ & $15(65.2)$ & $8(34.8)$ & $2.51(0.84-7.50)$ & 0.100 & $8(34.8)$ & $3.81(1.19-12.24)$ & 0.025 \\
\hline (+)DHEA, N (\%) & $6(100.0)$ & $0(0.0)$ & $\mathrm{NC}$ & NC & $0(0.0)$ & NC & NC \\
\hline$(+) \mathrm{GH}-\mathrm{DHEA}, \mathrm{N}(\%)$ & $17(77.3)$ & $5(22.7)$ & $1.38(0.41-4.63)$ & 0.599 & $5(22.7)$ & $2.10(0.59-7.50)$ & 0.253 \\
\hline \multicolumn{8}{|l|}{ Age $35-39$ years } \\
\hline$(-) \mathrm{GH}, \mathrm{N}(\%)$ & $86(90.5)$ & $9(9.5)$ & 1.00 & - & $2(2.1)$ & 1.00 & - \\
\hline$(+) \mathrm{GH}, \mathrm{N}(\%)$ & 34 (68.0) & $16(32.0)$ & $4.50(1.81-11.15)$ & 0.001 & $12(24.0)$ & 14.68 (3.14-68.76) & 0.001 \\
\hline (+)DHEA, N (\%) & $9(81.8)$ & $2(18.2)$ & $2.12(0.40-11.38)$ & 0.379 & $0(0.0)$ & $\mathrm{NC}$ & $\mathrm{NC}$ \\
\hline$(+) \mathrm{GH}-\mathrm{DHEA}, \mathrm{N}(\%)$ & $41(73.2)$ & $15(26.8)$ & $3.50(1.41-8.65)$ & 0.007 & $14(25.0)$ & 15.50 (3.37-71.28) & 0.000 \\
\hline \multicolumn{8}{|l|}{ Age $40-44$ years } \\
\hline$(-) \mathrm{GH}, \mathrm{N}(\%)$ & $78(94.0)$ & $5(6.0)$ & 1.00 & - & $2(2.4)$ & 1.00 & - \\
\hline$(+) \mathrm{GH}, \mathrm{N}(\%)$ & 69 (86.3) & $11(13.8)$ & $2.49(0.82-7.51)$ & 0.106 & $10(12.5)$ & $5.79(1.23-27.30)$ & 0.027 \\
\hline (+)DHEA, N (\%) & $22(95.7)$ & $1(4.3)$ & $0.71(0.08-6.39)$ & 0.759 & $0(0.0)$ & NC & NC \\
\hline$(+) \mathrm{GH}-\mathrm{DHEA}, \mathrm{N}(\%)$ & $84(89.4)$ & $10(10.6)$ & $1.86(0.61-5.67)$ & 0.277 & $5(5.3)$ & $2.28(0.43-12.05)$ & 0.334 \\
\hline \multicolumn{8}{|l|}{ Age 40-41 years } \\
\hline$(-) \mathrm{GH}, \mathrm{N}(\%)$ & $46(92.0)$ & $4(8.0)$ & 1.00 & - & $2(4.0)$ & 1.00 & - \\
\hline$(+) \mathrm{GH}, \mathrm{N}(\%)$ & $31(81.6)$ & $7(18.4)$ & $2.60(0.70-9.63)$ & 0.153 & $7(18.4)$ & $5.42(1.06-27.80)$ & 0.043 \\
\hline (+)DHEA, N (\%) & $10(90.9)$ & $1(9.1)$ & $1.15(0.12-11.42)$ & 0.905 & $0(0.0)$ & NC & NC \\
\hline (+)GH-DHEA, N (\%) & $29(87.9)$ & $4(12.1)$ & $1.59(0.37-6.84)$ & 0.536 & $3(9.1)$ & $2.40(0.38-15.21)$ & 0.353 \\
\hline
\end{tabular}

$\mathrm{Cl}$, confidence interval; DHEA, dehydroepiandrosterone; GH, growth hormone; NC, not computed due to low case number.

No pregnancies recorded over 44 years.

The positive effect of GH on clinical pregnancy or live birth chance was clearly dependent on patient age. Those younger than 39 years were more likely to achieve a live birth (+)GH than (-)GH. However, more beneficial GH effects were observed in the 35-39 years group as reflected by increased clinical pregnancy and live birth chance either in the absence or in the presence of DHEA. Limited GH benefits were observed for live birth in those aged 40-44 years and specifically were found in those aged 40 or 41 years.

\section{Analysis According to Transferred Embryo Quality and Adjuvant Treatment}

One of the most important independent predictors of pregnancy and live birth outcomes was the quality of the transferred embryo as assessed according to morphological grading (53). The majority of women/cycles $(90.1 \%)$ included the transfer of a day 3 cleavage stage embryo, whereas only 62 women/cycles $(9.9 \%)$ involved the transfer of a day 5 blastocyst, which is characteristic of a poor prognosis cohort (Table 5). Consequently, we focused on the interaction between transferred day 3 cleavage stage embryo quality and adjuvant treatment type (Table 5). High-quality day 3 embryos with $8+$ cells, no fragmentation and early compaction evident, led to greater pregnancy and live birth chance in comparison to low-quality day 3 embryos with slow cleavage and/or $>20 \%$ fragmentation, without evident compaction (Table 5). The (-) Adj group demonstrated greater pregnancy rates when highquality day 3 embryos were transferred. However, (+)GH with or without DHEA [i.e., (+)GH-DHEA] produced significantly more live births in comparison to (-)Adj and (+)DHEA when high-quality embryos were transferred (Table 5). Conversely, only $(+) \mathrm{GH}$ altered pregnancy and live birth rates when low-quality day 3 embryos were transferred (OR, 4.69; $95 \%$ CI, 1.43-15.37, $p=0.011$, and OR, 4.38 ; 95\% CI, 1.12-17.09, $p=0.034$, respectively). No other treatment group demonstrated increased clinical pregnancy rates with low-quality day 3 embryos (Table 5).

\section{DISCUSSION}

This retrospective study echoed our previous study (10) and showed that patient age, the quality of transferred embryos, and the utilization of GH (with or without DHEA) were significant predictors of clinical pregnancy and live birth rates in IVF patients categorized as poor prognosis. Importantly, following on from our previous study which focused solely on GH (10), the current investigation demonstrated that DHEA administration alone did not significantly influence primary outcomes in comparison to (-)Adj, and it did not alter nor enhance the positive effects from GH therapy when administered in combination. Generally, addition of DHEA to GH cycles led to a decrease in ORs, but the rates and ORs were still more beneficial in comparison to (-) Adj or (+)DHEA therapy alone. Furthermore, DHEA did not affect other secondary parameters such as number of oocytes 
TABLE 5 | Logistic regression analysis of transferred embryo quality interaction with $\mathrm{GH}$.

\begin{tabular}{|c|c|c|c|c|c|c|c|}
\hline & $\begin{array}{c}\text { No clinical } \\
\text { pregnancy, } \mathbf{n}(\%)\end{array}$ & $\begin{array}{c}\text { Yes clinical } \\
\text { pregnancy, } \mathbf{n}(\%)\end{array}$ & $\begin{array}{l}\text { Clinical pregnancy, } \\
\text { odds ratio }(95 \% \mathrm{Cl})\end{array}$ & $p$ value & $\begin{array}{c}\text { Yes live birth, } \\
\text { n (\%) }\end{array}$ & $\begin{array}{l}\text { Live birth, odds } \\
\text { ratio }(95 \% \mathrm{Cl})\end{array}$ & $p$ value \\
\hline \multicolumn{8}{|l|}{ Unadjusted analysis } \\
\hline$(-) \mathrm{GH}, \mathrm{N}(\%)$ & $215(90.0)$ & $24(10.0)$ & 1.00 & - & $11(4.6)$ & 1.00 & - \\
\hline$(+) \mathrm{GH}, \mathrm{N}(\%)$ & $126(78.3)$ & $35(21.7)$ & $2.49(1.42-4.37)$ & 0.002 & 30 (18.6) & $4.75(2.30-9.79)$ & 0.000 \\
\hline (+)DHEA, N (\%) & 39 (92.8) & $3(7.1)$ & $0.69(0.20-2.40)$ & 0.559 & $0(0.0)$ & $\mathrm{NC}$ & NC \\
\hline$(+) \mathrm{GH}-\mathrm{DHEA}, \mathrm{N}(\%)$ & $154(83.7)$ & $30(16.3)$ & $1.75(0.98-3.10)$ & 0.058 & $24(13.0)$ & $3.11(1.48-6.53)$ & 0.003 \\
\hline \multicolumn{8}{|c|}{ Analysis According to transferred embryo quality } \\
\hline \multicolumn{8}{|c|}{ High-quality day 3 embryo } \\
\hline$(-) \mathrm{GH}, \mathrm{N}(\%)$ & $87(86.1)$ & $14(13.9)$ & 1.00 & - & $7(6.9)$ & 1.00 & - \\
\hline$(+) \mathrm{GH}, \mathrm{N}(\%)$ & $57(77.0)$ & $17(23.0)$ & $1.85(0.85-4.05)$ & 0.122 & $16(21.6)$ & $3.70(1.44-9.55)$ & 0.007 \\
\hline (+)DHEA, N (\%) & $18(100.0)$ & $0(0.0)$ & $\mathrm{NC}$ & NC & $0(0.0)$ & $\mathrm{NC}$ & NC \\
\hline$(+) \mathrm{GH}-\mathrm{DHEA}, \mathrm{N}(\%)$ & $47(81.0)$ & $11(19.0)$ & $1.45(0.61-3.46)$ & 0.396 & $10(17.2)$ & $2.80(1.00-7.81)$ & 0.050 \\
\hline \multicolumn{8}{|c|}{ Low-quality day 3 embryo } \\
\hline$(-) \mathrm{GH}, \mathrm{N}(\%)$ & $104(96.3)$ & $4(3.7)$ & 1.00 & - & $3(2.8)$ & 1.00 & - \\
\hline$(+) \mathrm{GH}, \mathrm{N}(\%)$ & $61(84.7)$ & $11(15.3)$ & $4.69(1.43-15.37)$ & 0.011 & $8(11.1)$ & $4.38(1.12-17.09)$ & 0.034 \\
\hline (+)DHEA, N (\%) & $18(85.7)$ & $3(14.3)$ & $4.33(0.89-21.00)$ & 0.069 & $0(0.0)$ & $\mathrm{NC}$ & NC \\
\hline$(+) \mathrm{GH}-\mathrm{DHEA}, \mathrm{N}(\%)$ & $100(89.3)$ & $12(10.7)$ & $3.12(0.97-10.00)$ & 0.055 & $7(6.3)$ & $2.33(0.59-9.27)$ & 0.229 \\
\hline
\end{tabular}

$\mathrm{Cl}$, confidence interval; NC, not computed due to low case number.

The positive effect of GH on clinical pregnancy or live birth chance was clearly dependent on the quality of transferred embryos. (+)GH increased the clinical pregnancy as well as live birth chance when lower quality Day-3 embryos were transferred, but only improved live birth chance when high quality Day-3 embryos were transferred. However, GH in combination with DHEA had little impact on these outcomes.

retrieved or fertilization rates as previously reported $(27,32$, $35,36)$. Contrary to other reports $(25-34)$, these findings question the use of DHEA as an adjuvant in IVF, but reiterate the potential beneficial effects of $\mathrm{GH}$.

While we acknowledge that the (+)DHEA alone group contained the smallest number of cases, the evidence suggested that DHEA did not convey any beneficial effect as an IVF adjuvant, including with $\mathrm{GH}$, and these data build upon the only other report to investigate the impact of DHEA and GH combinations in IVF (45). In their study of 85 women receiving 183 IVF cycles, Haydardedeoğlu et al. demonstrated that DHEA for 12 weeks, with at least 4 weeks of transdermal testosterone and a late-luteal administration of $\mathrm{GH}$ in the previous cycle prior to stimulation, resulted in greater clinical pregnancy and live birth rates, along with more follicles, oocytes retrieved, and higher fertilization rate. There was no difference reported in the morphological quality of developing embryos (45). In our analysis, we also observed differences in pregnancy and live birth rates (45). We also found no significant change in the number of oocytes retrieved nor the fertilization rate. The number of zygotes with two pronuclei developing was lower with (+)GH-DHEA, but not significant, although it may reflect the significantly increased age of the women undertaking these combination adjuvant cycles in our study. Similar to the aforementioned study, we also observed no significant difference in embryo quality as assessed by the morphological analysis. However, they used a complicated treatment strategy that involved three different adjuvant agents, and the treatment group also consisted of only 37 cycles (45). Therefore, our combination study may indeed be considered superior to the study by Haydardedeoğlu et al., until the data become available for the combined GH-DHEA trial NCT02766764, which is currently recruiting.
Importantly, the $(+) \mathrm{GH}$ results in this study also reflect our earlier work (13) and comparable data derived from an RCT by Tesarik et al. (16), which suggested that GH lowered miscarriage rate leading to more live births. There are some notable differences between the studies such as patients in the study by Tesarik et al. being older with a mean age of 42 years and the researchers transferring three to four embryos. In terms of the ORs for clinical pregnancy and live births in this study, similar ORs were observed in several meta-analyses $(12,15,17)$. However, other recent meta-analyses indicated that $\mathrm{GH}$ had no significant influence on live birth, with lower ORs $(7,8)$, but a slight increase in pregnancy rate (8).

The main aim of this study is to retrospectively examine in one of the largest adjuvant cohorts, the impact of adjuvant therapy on clinical IVF outcomes. Another crucial aim is to identify critical confounding variables and participant inclusion characteristics outside of the Bologna criteria that could be considered when designing future IVF adjuvant RCTs. The most important confounding variables we identified were patient age and quality of the transferred embryo, which is supported by our previous report (10). In this analysis, we showed that additional patient characteristics such as $\mathrm{AMH}, \mathrm{BMI}$, number of infertility factors, previous IVF attempts, or mean number of embryos transferred did not have an independent effect on clinical pregnancy or live birth chance and may be related to our specific $\mathrm{rFSH}$ dosing algorithms $(48,54)$.

The patients in our analysis were categorized as poor prognosis, with low AFC ( $\leq 8$ follicles) and significantly reduced serum AMH level. Those treated with $(+) \mathrm{GH},(+)$ DHEA, and $(+) \mathrm{GH}-\mathrm{DHEA}$ were older on average with a lower $\mathrm{AMH}$ in comparison to those (-)Adj. In spite of this perceived very poor ovarian reserve and advanced maternal age, we showed that when $(+) \mathrm{GH}$ was added to cycles, with or without DHEA, the 
oocyte and embryo utilization rates, along with pregnancy and live births rates, were greater. We also investigated the effect of (+) GH on patients with different AFC gradings, but neither AFC nor the presence or absence of GH or DHEA significantly altered clinical pregnancy or live birth chance in different AFC groupings. Notably, because patient ovarian reserve has not been described in any other IVF study utilizing GH (17), direct comparison of our findings is restricted. However, AFC as a specific marker of ovarian reserve was insignificant following adjustment for patient age. In relation to age, we did not observe any benefit in patients older than 41 years. Taken together, these findings have implications for these specific parameters within the Bologna criteria. Therefore, newer adjuvant studies should probably focus on women 40 years or below, who generate lower quality embryos with standard stimulation and with RIF, rather than those defined by the Bologna criteria.

There are significant strengths associated with this study including the use of a large data set ( $n=626$ cycles), a low number of transferred embryos per cycle (average 1.5/cycle), and incorporation of several potential confounding variables such as age, AFC, AMH, and transferred embryo quality. However, like all retrospective analyses and most $\mathrm{GH}$ and DHEA studies in the IVF research space, it has significant limitations that should be cautiously considered when interpreting the findings. The potential positive effects of GH and the disappointing effects of DHEA are strictly associative rather than causative, as this study was not designed as an intervention RCT. In addition, given the retrospective design, the study is also subject to significant patient selection bias and the process by which the control group [i.e., (-)Adj] was selected must be carefully understood. Justifiably, it was decided to generate this group using treatment cycles free from adjuvant intervention, but to only include these adjuvant-free cycles from women who received adjuvant therapy during their entire treatment history. This meant that the group comprised only women considered poor prognosis, being offered adjuvant therapy due to one or more of the five reasons outlined in Section "Materials and Methods." In an attempt to randomize cases and to counter any other perceived selection bias, we chose to analyze only the very first IVF cycle with fresh embryo transfer for each women during the study period, regardless of whether there was an adjuvant intervention. This process also prevented any confounding that may be interpreted from including multiple treatment cycles for individual women.

In addition, there is a significant heterogeneity in terms of poor prognosis factors and combination thereof in the adjuvant treatment groups. While the adjuvant groups, especially $\mathrm{GH}$, tended to have a higher proportion of patients with these factors, the heterogeneity is an important limitation of this study. Future studies must focus on more homogenous groups, but as indicated here, these could incorporate factors outside of the traditional Bologna criteria and possibly have a more nuanced approach like that of the POSEIDON study (55). Other factors that may be important but not considered in the current analysis was patient socioeconomic status and parity. Socioeconomic status and affordability could be critical confounders as patients were required to pay for IVF adjuvants. Taken together, while not as robustly designed and as powerful as a prospective RCT, the current study design limited any perceived bias and is one of the largest GH studies in IVF research to date.

Overall, we have shown for the first time in a large data set, and adjusting for various confounding variables, that $(+) \mathrm{GH}$ alone or in combination with DHEA, significantly increased the chance of clinical pregnancy and live birth outcomes in poor prognosis IVF patients, who tended to be significantly older (1.5-2.0 years) and had a lower average serum AMH value. This new retrospective adjuvant IVF study with GH and DHEA alone and in combination is the first to include aspects of analysis such as AFC, AMH, BMI, and embryo quality assessment and has provided further evidence to indicate the potential beneficial effects of GH supplementation in IVF treatment. It also demonstrated that DHEA did not appear to have a significant influence on primary IVF outcomes. Although the study has certain limitations in that it is observational and retrospective in nature, bias was minimized as best as possible, and the data suggested that GH supplementation may provide more live births, mainly in younger women and questions the use of adjuvant therapy in women older than 41 years. Finally, the data indicate that future GH and DHEA IVF RCTs should strictly account for transferred embryo quality and expectedly age, while possibly focusing on younger women with RIF or those generating a greater proportion of low-quality embryos.

\section{ETHICS STATEMENT}

Our clinic is accredited with the Reproductive Technology Accreditation Committee, a National body, as well as the Reproductive Technology Council of Western Australia, a State body. These agencies monitor all activities according to respective Codes of Practice. Specific ethics approval was not required for this study as all procedures and blood tests were embraced by routine approved clinical protocols. However, retrospective analysis and reporting of the data was approved under Curtin University Ethics Committee approval no. RD_25-10. In addition, as a part of our documentation system, written informedconsent was obtained from each participant who accepted the use of adjuvants, and they were required to pay for these adjuvants over and above the IVF treatment charges.

\section{AUTHOR CONTRIBUTIONS}

The present work was designed by JY, KK, and SD. Data extraction and analysis were performed by KK, PH, SD, and JY. Patient recruitment was undertaken by $\mathrm{PR}, \mathrm{GB}$, and SS. The initial manuscript draft was prepared by KK and subsequently revised by JY, $\mathrm{SD}$, and $\mathrm{PH}$. All the authors approved the final submitted version.

\section{ACKNOWLEDGMENTS}

The authors thank Curtin University Faculty of Health Science and PIVET Medical Centre for research support. All staff and research participants at PIVET Medical Centre are acknowledged with thanks. 


\section{REFERENCES}

1. Ferraretti AP, La Marca A, Fauser BC, Tarlatzis B, Nargund G, Gianaroli L, et al. ESHRE consensus on the definition of 'poor response' to ovarian stimulation for in vitro fertilization: the Bologna criteria. Hum Reprod (2011) 26:1616-24. doi:10.1093/humrep/der092

2. Nardo LG, El-Toukhy T, Stewart J, Balen AH, Potdar N. British Fertility Society Policy and Practice Committee: adjuvants in IVF: evidence for good clinical practice. Hum Fertil (2015) 18:2-15. doi:10.3109/14647273. 2015.985454

3. Homburg R, Eshel A, Abdalla HI, Jacobs HS. Growth hormone facilitates ovulation induction by gonadotrophins. Clin Endocrinol (Oxf) (1988) 29: 113-7. doi:10.1111/j.1365-2265.1988.tb00252.x

4. Busacca M, Fusi FM, Brigante C, Bonzi V, Gonfiantini C, Vignali M, et al. Use of growth hormone-releasing factor in ovulation induction in poor responders. J Reprod Med (1996) 41:699-703.

5. Bassiouny YA, Dakhly DMR, Bayoumi YA, Hashish NM. Does the addition of growth hormone to the in vitro fertilization/intracytoplasmic sperm injection antagonist protocol improve outcomes in poor responders? A randomized, controlled trial. Fertil Steril (2015). doi:10.1016/j. fertnstert.2015.11.026

6. Bayoumi YA, Dakhly DMR, Bassiouny YA, Hashish NM. Addition of growth hormone to the microflare stimulation protocol among women with poor ovarian response. Int J Gynaecol Obstet (2015) 131:305-8. doi:10.1016/j. ijgo.2015.05.034

7. Yu X, Ruan J, He L-P, Hu W, Xu Q, Tang J, et al. Efficacy of growth hormone supplementation with gonadotrophins in vitro fertilization for poor ovarian responders: an updated meta-analysis. Int J Clin Exp Med (2015) 8:4954-67.

8. Hart RJ, Rombauts L, Norman RJ. Growth hormone in IVF cycles: any hope? Curr Opin Obstet Gynecol (2017) 29:119-25. doi:10.1097/ GCO.0000000000000360

9. Li X-L, Wang L, Lv F, Huang X-M, Wang L-P, Pan Y, et al. The influence of different growth hormone addition protocols to poor ovarian responders on clinical outcomes in controlled ovary stimulation cycles: a systematic review and meta-analysis. Medicine (2017) 96:e6443. doi:10.1097/MD. 0000000000006443

10. Keane KN, Yovich JL, Hamidi A, Hinchliffe PM, Dhaliwal SS. Single-centre retrospective analysis of growth hormone supplementation in IVF patients classified as poor-prognosis. BMJ Open (2017) 7:e018107. doi:10.1136/ bmjopen-2017-018107

11. Kolibianakis EM, Venetis CA, Diedrich K, Tarlatzis BC, Griesinger G. Addition of growth hormone to gonadotrophins in ovarian stimulation of poor responders treated by in-vitro fertilization: a systematic review and meta-analysis. Hum Reprod Update (2009) 15:613-22. doi:10.1093/humupd/ dimp026

12. Kyrou D, Kolibianakis EM, Venetis CA, Papanikolaou EG, Bontis J, Tarlatzis BC. How to improve the probability of pregnancy in poor responders undergoing in vitro fertilization: a systematic review and meta-analysis. Fertil Steril (2009) 91:749-66. doi:10.1016/j.fertnstert.2007.12.077

13. Yovich JL, Stanger JD. Growth hormone supplementation improves implantation and pregnancy productivity rates for poor-prognosis patients undertaking IVF. Reprod Biomed Online (2010) 21:37-49. doi:10.1016/j.rbmo. 2010.03.013

14. Lattes K, Brassesco M, Gomez M, Checa MA. Low-dose growth hormone supplementation increases clinical pregnancy rate in poor responders undergoing in vitro fertilisation. Gynecol Endocrinol (2015) 31:565-8. doi:10.3109/ 09513590.2015 .1025378

15. Jeve YB, Bhandari HM. Effective treatment protocol for poor ovarian response: a systematic review and meta-analysis. J Hum Reprod Sci (2016) 9:70-81. doi:10.4103/0974-1208.183515

16. Tesarik J, Hazout A, Mendoza C. Improvement of delivery and live birth rates after ICSI in women aged $>40$ years by ovarian co-stimulation with growth hormone. Hum Reprod (2005) 20:2536-41. doi:10.1093/humrep/dei066

17. Duffy JM, Ahmad G, Mohiyiddeen L, Nardo LG, Watson A. Growth hormone for in vitro fertilization. Cochrane Database Syst Rev (2010) 1:CD000099. doi:10.1002/14651858.CD000099

18. Owen EJ, West C, Mason BA, Jacobs HS. Co-treatment with growth hormone of sub-optimal responders in IVF-ET. Hum Reprod (1991) 6:524-8. doi:10.1093/oxfordjournals.humrep.a137372
19. Levy T, Limor R, Villa Y, Eshel A, Eckstein N, Vagman I, et al. Another look at co-treatment with growth hormone and human menopausal gonadotrophins in poor ovarian responders. Hum Reprod (1993) 8:834-9. doi:10.1093/ oxfordjournals.humrep.a138150

20. Bergh C, Hillensjo T, Wikland M, Nilsson L, Borg G, Hamberger L. Adjuvant growth hormone treatment during in vitro fertilization: a randomized, placebo-controlled study. Fertil Steril (1994) 62:113-20. doi:10.1016/S00150282(16)56825-3

21. Dor J, Seidman DS, Amudai E, Bider D, Levran D, Mashiach S. Adjuvant growth hormone therapy in poor responders to in-vitro fertilization: a prospective randomized placebo-controlled double-blind study. Hum Reprod (1995) 10:40-3. doi:10.1093/humrep/10.1.40

22. Suikkari A, Maclachlan V, Koistinen R, Seppala M, Healy D. Doubleblind placebo controlled study: human biosynthetic growth hormone for assisted reproductive technology. Fertil Steril (1996) 65:800-5. doi:10.1016/ S0015-0282(16)58217-X

23. Eftekhar M, Aflatoonian A, Mohammadian F, Eftekhar T. Adjuvant growth hormone therapy in antagonist protocol in poor responders undergoing assisted reproductive technology. Arch Gynecol Obstet (2013) 287:1017-21. doi:10.1007/s00404-012-2655-1

24. Arlt W. Dehydroepiandrosterone and ageing. Best Pract Res Clin Endocrinol Metab (2004) 18:363-80. doi:10.1016/j.beem.2004.02.006

25. Casson PR, Lindsay MS, Pisarska MD, Carson SA, Buster JE. Dehydroepiandrosterone supplementation augments ovarian stimulation in poor responders: a case series. Hum Reprod (2000) 15:2129-32. doi:10.1093/ humrep/15.10.2129

26. Barad D, Brill H, Gleicher N. Update on the use of dehydroepiandrosterone supplementation among women with diminished ovarian function. J Assist Reprod Genet (2007) 24:629-34. doi:10.1007/s10815-007-9178-x

27. Gleicher N, Weghofer A, Barad D. Dehydroepiandrosterone (DHEA) reduces embryo aneuploidy: direct evidence from preimplantation genetic screening (PGS). Reprod Biol Endocrinol (2010) 8:140. doi:10.1186/14777827-8-140

28. Wiser A, Gonen O, Ghetler Y, Shavit T, Berkovitz A, Shulman A. Addition of dehydroepiandrosterone (DHEA) for poor-responder patients before and during IVF treatment improves the pregnancy rate: a randomized prospective study. Hum Reprod (2010) 25:2496-500. doi:10.1093/humrep/deq220

29. Gleicher N, Barad D. Dehydroepiandrosterone (DHEA) supplementation in diminished ovarian reserve (DOR). Reprod Biol Endocrinol (2011) 9:1-12. doi:10.1186/1477-7827-9-67

30. Xu B, Li Z, Yue J, Jin L, Li Y, Ai J, et al. Effect of dehydroepiandrosterone administration in patients with poor ovarian response according to the Bologna criteria. PLoS One (2014) 9:e99858. doi:10.1371/journal.pone. 0099858

31. Tartagni M, Cicinelli MV, Baldini D, Tartagni MV, Alrasheed H, Desalvia MA, et al. Dehydroepiandrosterone decreases the age-related decline of the in vitro fertilization outcome in women younger than 40 years old. Reprod Biol Endocrinol (2015) 13:18. doi:10.1186/s12958-015-0014-3

32. Kotb MMM, Hassan AMA, Awadallah AMA. Does dehydroepiandrosterone improve pregnancy rate in women undergoing IVF/ICSI with expected poor ovarian response according to the Bologna criteria? A randomized controlled trial. Eur JObstet Gynecol Reprod Biol (2016) 200:11-5. doi:10.1016/j. ejogrb.2016.02.009

33. Liu X, Wang W, Wang X, Qu Q, Hao C. Effect of dehydroepiandrosterone administration in Chinese women over 37 years undergoing assisted reproductive techniques. Eur J Obstet Gynecol Reprod Biol (2016) 202:9-13. doi:10.1016/j.ejogrb.2016.04.028

34. Zhang M, Niu W, Wang Y, Xu J, Bao X, Wang L, et al. Dehydroepiandrosterone treatment in women with poor ovarian response undergoing IVF or ICSI: a systematic review and meta-analysis. J Assist Reprod Genet (2016) 33:981-91. doi:10.1007/s10815-016-0713-5

35. Barad D, Gleicher N. Effect of dehydroepiandrosterone on oocyte and embryo yields, embryo grade and cell number in IVF. Hum Reprod (2006) 21:2845-9. doi:10.1093/humrep/del254

36. Zhang HH, Xu PY, Wu J, Zou WW, Xu XM, Cao XY, et al. Dehydroepiandrosterone improves follicular fluid bone morphogenetic protein15 and accumulated embryo score of infertility patients with diminished ovarian reserve undergoing in vitro fertilization: a randomized controlled trial. J Ovarian Res (2014) 7:93. doi:10.1186/s13048-014-0093-3 
37. Sunkara SK, Pundir J, Khalaf Y. Effect of androgen supplementation or modulation on ovarian stimulation outcome in poor responders: a meta-analysis. Reprod Biomed Online (2011) 22:545-55. doi:10.1016/j. rbmo.2011.01.015

38. Narkwichean A, Maalouf W, Campbell B, Jayaprakasan K. Efficacy of dehydroepiandrosterone to improve ovarian response in women with diminished ovarian reserve: a meta-analysis. Reprod Biol Endocrinol (2013) 11:44. doi:10.1186/1477-7827-11-44

39. Qin JC, Fan L, Qin AP. The effect of dehydroepiandrosterone (DHEA) supplementation on women with diminished ovarian reserve (DOR) in IVF cycle: evidence from a meta-analysis. J Gynecol Obstet Hum Reprod (2017) 46:1-7. doi:10.1016/j.jgyn.2016.01.002

40. Motta EL, Rossi LM, Fernandes TR, Massaguer AA, Fassolas G, Serafini P. The use of DHEA in poor responders does not improve IVF outcomes: insights of a pilot study. Fertil Steril (2006) 86:S428. doi:10.1016/j.fertnstert. 2006.07.1183

41. Moawad A, Shaeer M. Long-term androgen priming by use of dehydroepiandrosterone (DHEA) improves IVF outcome in poor-responder patients. A randomized controlled study. Middle East Fertil Soc J (2012) 17:268-74. doi:10.1016/j.mefs.2012.11.002

42. Vlahos N, Papalouka M, Triantafyllidou O, Vlachos A, Vakas P, Grimbizis G, et al. Dehydroepiandrosterone administration before IVF in poor responders: a prospective cohort study. Reprod Biomed Online (2015) 30:191-6. doi:10.1016/j.rbmo.2014.10.005

43. Yeung TWY, Chai J, Li RHW, Lee VCY, Ho PC, Ng EHY. A double-blind randomised controlled trial on the effect of dehydroepiandrosterone on ovarian reserve markers, ovarian response and number of oocytes in anticipated normal ovarian responders. BJOG (2016) 123:1097-105. doi:10.1111/ 1471-0528.13808

44. Weil S, Vendola K, Zhou J, Bondy CA. Androgen and follicle-stimulating hormone interactions in primate ovarian follicle development. J Clin Endocrinol Metab (1999) 84:2951-6. doi:10.1210/jcem.84.8.5929

45. Haydardedeoğlu B, Işık AZ, Bulgan Kılıçdağ E. The combination of dehydroepiandrosterone, transdermal testosterone, and growth hormone as an adjuvant therapy in assisted reproductive technology cycles in patients aged below 40 years with diminished ovarian reserve. Turk J Obstet Gynecol (2015) 12:60-5. doi:10.4274/tjod.32656

46. Yovich JL, Stanger JD, Yovich JM, Tuvik AI. Quality of embryos from in-vitro fertilisation. Lancet (1984) 1:457. doi:10.1016/S0140-6736(84)91794-X

47. Keane KN, Hinchliffe PM, Namdar N, Conceicao JL, Newsholme P, Yovich JL. Novel dehydroepiandrosterone troche supplementation improves the serum androgen profile of women undergoing in vitro fertilization. Drug Des Devel Ther (2015) 9:5569-78. doi:10.2147/DDDT.S92467

48. Yovich JL, Alsbjerg B, Conceicao JL, Hinchliffe PM, Keane KN. PIVET rFSH dosing algorithms for individualized controlled ovarian stimulation enables optimized pregnancy productivity rates and avoidance of ovarian hyperstimulation syndrome. Drug Des Devel Ther (2016) 10:256173. doi:10.2147/DDDT.S104104

49. Fisch JD, Keskintepe L, Sher G. Gonadotropin-releasing hormone agonist/ antagonist conversion with estrogen priming in low responders with prior in vitro fertilization failure. Fertil Steril (2008) 89:342-7. doi:10.1016/j. fertnstert.2007.03.004

50. Yovich JL, Stanger JD, Yovich JM, Tuvik AI. Assessment and hormonal treatment of the luteal phase of in vitro fertilization cycles. Aust $N$ Z J Obstet Gynaecol (1984) 24:125-30. doi:10.1111/j.1479-828X.1984. tb01472.x

51. Yovich JL, Grudzinskas G. In vitro fertilization and embryo transfer (IVF-ET): current status. The Management of Infertility, a Manual of Gamete Handling Procedures. (Chap. 10), Oxford. UK: Heinemann Medical Books (1990). p. 121-44.

52. Gardner DK, Schoolcraft WB. In vitro culture of human blastocysts. Towards Reprod Certainty (1999) 1999:378-88

53. Yovich JL, Conceicao JL, Stanger JD, Hinchliffe PM, Keane KN. Midluteal serum progesterone concentrations govern implantation rates for cryopreserved embryo transfers conducted under hormone replacement. Reprod Biomed Online (2015) 31:180-91. doi:10.1016/j.rbmo. 2015.05.005

54. Yovich J, Stanger J, Hinchliffe P. Targeted gonadotrophin stimulation using the PIVET algorithm markedly reduces the risk of OHSS. Reprod Biomed Online (2012) 24:281-92. doi:10.1016/j.rbmo.2011.11.014

55. Humaidan P, Alviggi C, Fischer R, Esteves SC. The novel POSEIDON stratification of 'Low prognosis patients in Assisted Reproductive Technology' and its proposed marker of successful outcome. F1000Res (2016) 5:2911. doi:10.12688/f1000research.10382.1

Conflict of Interest Statement: All authors have nothing to disclose and approve the submitted copy of the manuscript.

KK is employed as a Research Fellow at Curtin University and a portion of this position is financially supported by PIVET Medical Centre. PH, PR, GB, and SS provide consultancy services to PIVET Medical Centre. SD is a Curtin University Professor and an adjunct Research Consultant at PIVET Medical Centre. JY is the salaried Medical Director of PIVET Medical Centre and an adjunct Clinical Professor at Curtin University.

Copyright (c) 2018 Keane, Hinchliffe, Rowlands, Borude, Srinivasan, Dhaliwal and Yovich. This is an open-access article distributed under the terms of the Creative Commons Attribution License (CC BY). The use, distribution or reproduction in other forums is permitted, provided the original author(s) and the copyright owner are credited and that the original publication in this journal is cited, in accordance with accepted academic practice. No use, distribution or reproduction is permitted which does not comply with these terms. 